\title{
Analysis on the Governing Reactions in Coal Oxidation at Temperatures up to $400^{\circ} \mathrm{C}$
}

\author{
Jing Zhan, Haihui Wang*, Feng Zhu, Shengnan Song \\ State Key Laboratory of Fire Science, University of Science and Technology of China, Hefei, China \\ Email: "hhwang4@ustc.edu.cn
}

Received 17 March 2014; revised 21 April 2014; accepted 23 May 2014

Copyright (c) 2014 by authors and Scientific Research Publishing Inc.

This work is licensed under the Creative Commons Attribution International License (CC BY).

http://creativecommons.org/licenses/by/4.0/

(c) (i) Open Access

\begin{abstract}
The present study aims to further understanding of the principal reactions that occur during coal oxidation at moderate temperatures. Mass change and heat evolution of a sample were monitored by thermo-gravimetric analysis coupled with differential thermal analysis (TGA/DTA). Gaseous and solid products were traced using online or in situ Fourier transform infrared spectroscopy (FTIR). Measurements were conducted by heating the samples up to $400^{\circ} \mathrm{C}$, with the $\mathrm{O}_{2}$ concentration in the reaction medium set at $0,10,21$, and $40 \mathrm{vol} \%$, respectively. It was observed that the mass increase of a sample between $150^{\circ} \mathrm{C}$ and $\sim 275^{\circ} \mathrm{C}$ was a result of the accumulation of $\mathrm{C}=\mathrm{O}$ containing species in the coal structure, whereas substantial mass loss and heat evolution of a sample at $\sim 400^{\circ} \mathrm{C}$ can be attributed to the significant involvement of the direct "burn-off" reaction. Enrichment of $\mathrm{O}_{2}$ in the reaction medium leads to the acceleration in oxygen chemisorption, formation and decomposition of the solid oxygenated complexes, as well as the "burn-off" reaction. With the temperature increasing, the oxidation process governed by oxygen chemisorption gradually shifts to that by significant decomposition reactions, and eventually to that by the direct "burn-off" reaction. Temperature boundaries of these stages can be determined using parameters defined based on a set of TG/DTA data. Shift in the governing reactions is essentially due to the diverse requirements of reactants of the reactions and their energy barriers to be overcome. In engineering practice, the phenomena of self-heating and spontaneous combustion of coal correspond to chemisorption and the direct "burn-off" reaction, respectively.
\end{abstract}

Keywords: Coal; Oxidation at Moderate Temperatures; Reaction Product; Governing Reaction; Self-Heating; Spontaneous Combustion

\section{Introduction}

Coal oxidation at moderate temperatures $\left(\leq 400^{\circ} \mathrm{C}\right)$ alters the coal properties as fuel, thereby making pronounced impact on its subsequent utilization [1] [2]. As an exothermic phenomenon, coal oxidation has direct link to self-heating and spontaneous combustion of coal, which poses fire hazard in coal industries. Up to date, enormous efforts have been made to understand the chemistry and heat effects of the oxidation process [1]-[4].

Coal oxidation at moderate temperatures is a complicated process affected by several factors, including coal compositions, temperature and oxygen concentration in the environment [1]-[3]. As stated in the literature [2] [3]

"Corresponding author. the rate of oxygen consumption almost doubles by every increment of $10^{\circ} \mathrm{C}$ at temperatures below $90^{\circ} \mathrm{C}$. At a specific temperature, an oxygen-enriched environment may result in acceleration in the oxidation rate, thereby producing a larger amount of heat during this process.

In the past two decades, typical research work on the effect of temperature on coal oxidation can be attributed to the observations based on the thermo-gravimetric analysis (TGA) and associated techniques. Crelling and coworkers [5] monitored the temperature-dependent mass change of a series of coals oxidized in the aerial atmosphere, and then suggested several temperature parameters to describe the profile of the TG curves collected. The parameters below $400^{\circ} \mathrm{C}$ include "temperature of initial chemisorptions" $T_{1}$, "temperature of maximum rate of

How to cite this paper: Zhan, J., Wang, H.H., Zhu, F. and Song, S.N. (2014) Analysis on the Governing Reactions in Coal Oxidation at Temperatures up to $400^{\circ} \mathrm{C}$. International Journal of Clean Coal and Energy, 3, 19-28.

http://dx.doi.org/10.4236/ijcce.2014.32003 
chemisorptions" $T_{2}$, and "temperature of combustion onset” $T_{3}$. Based on these temperature parameters, He and Wang [6] proposed four stages of the coal oxidation at low and moderate temperatures, i.e., moisture vaporization, mass increase by absorbing oxygen, degradation caused by heating, and combustion. The rate of mass increase at the second stage was defined and then utilized to evaluate the propensity of various coals towards spontaneous combustion [6] [7].

So far, significant progress has been made in understanding the mechanism of coal oxidation. It has been identified that two independent reaction sequences pro-

ceed during the oxidation process, and one of them is the chemisorption sequence [2] [4] [8]. Chemisorption sequence consists of several steps, including the chemisorption of oxygen, as well as formation and decomposetion of unstable carbon-oxygen complexes (intermediates). The other reaction sequence is the so-called direct "burnoff” reaction, which occurs at specific sites in the coal structure. Given that the intermediate process is significantly rapid, direct "burn-off" reaction is described as a simultaneous formation of the gas products, i.e., $\mathrm{CO}_{2}$, $\mathrm{CO}$, and $\mathrm{H}_{2} \mathrm{O}$. These two reaction sequences are briefly depicted as follows:

Gas species

Unstable solid complexes

Stable solid complexes

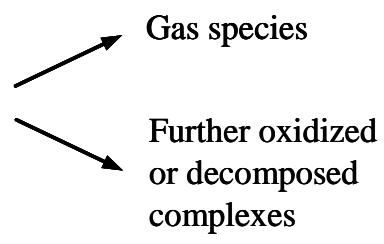

Although the occurrence of the direct "burn-off" reaction at low temperatures $\left(<150^{\circ} \mathrm{C}\right)$ has been verified by the existing measurements and theoretical analysis [2] [4] [8] [9], the dependence of this reaction to temperature and its relationship with the chemisorption sequence have not been clearly elucidated. In addition, extensive measurements have been conducted to verify the effect of $\mathrm{O}_{2}$ concentration on coal oxidation; however, no rigorous efforts have been made to understand the role of $\mathrm{O}_{2}$ molecules in altering the multi-step reactions that occur during the oxidation process.

The present study aims to further understanding of the principal reactions that occur during coal oxidation at moderate temperatures and lay a methodical basis for developing new techniques to characterize the coals and to assess their propensity towards self-heating and spontaneous combustion. Using TGA and differential thermal analysis (DTA) coupling technique, the mass change and heat evolution are measured during coal oxidation under various oxygen concentrations and at temperatures up to $400^{\circ} \mathrm{C}$. Gas and solid products formed on coal surfaces are also monitored by means of a TGA in connection with a Fourier transform infrared spectrometer (TGA/ FTIR), as well as an in-situ FTIR technique. The nature of different reactions and their inherent relationships are then explored based on a series of experimental results obtained.

\section{Experimental}

A bituminous coal was collected from Yanzhou Colliery,
Shandong Province. The coal was crushed and ground into particles. Size distribution of the particles was measured using laser particle-size-distribution tester JL-1177, and the volumetric mean diameter of the particles $(\mathrm{D}[\mathrm{v}$, $0.5]$ ) was found to be $\sim 1.6 \mu \mathrm{m}$. Fine coal particles were used to eliminate the possible reaction regime controlled by gas diffusion in coal pores during the oxidation process [2] [10]. Proximate and ultimate analyses of the coal are given in Table 1.

A TGA/DTA instrument was used to evaluate pyrolysis and oxidation behaviors of raw coal samples, and the one adopted was a Shimadzu DTG-60H thermal analysis system with a nominal resolution of mass of $0.1 \mu \mathrm{g}$. DTA signals at a sensitivity of $0.1 \mu \mathrm{V}$ were directly converted into heat flow rates in the units of $\mathrm{mW}$ per sample current mass using a manufacturer-provided software. A sample of $\sim 10 \mathrm{mg}$ in mass was quickly and loosely placed on the aluminum crucible. The sample temperature was then raised from room temperature to $400^{\circ} \mathrm{C}$ at a heating rate of $4^{\circ} \mathrm{C} / \mathrm{min}$. Such a low heating rate employed was to reduce the thermal lag of a sample during heating.

Table 1. Proximate and ultimate analyses for the coal used in the current study.

\begin{tabular}{cclc}
\hline $\begin{array}{c}\text { Proximate analysis } \\
\text { (air-dry basis) }\end{array}$ & & \multicolumn{2}{c}{$\begin{array}{c}\text { Ultimate analysis } \\
\text { (dry ash free basis) }\end{array}$} \\
\hline Moisture (\%) & 2.8 & $\mathrm{C} \mathrm{( \% )}$ & 83.5 \\
Ash (\%) & 13.1 & $\mathrm{H} \mathrm{( \% )}$ & 5.0 \\
Volatile matter (\%) & 32.5 & $\mathrm{~N} \mathrm{( \% )}$ & 1.2 \\
Fixed carbon (\%) & 51.1 & $\mathrm{~S} \mathrm{( \% )}$ & 0.6 \\
Total sulfur (\%) & 0.5 & $\mathrm{O} \mathrm{( \% )}$ & 9.7 \\
\hline
\end{tabular}


Flow rate of the atmosphere purging into the furnace was set at $50 \mathrm{~cm}^{3} / \mathrm{min}$. Industrial-grade nitrogen gas was used in the pyrolysis experiment, and the oxidation measurements of the samples were performed in atmospheres that contain $\mathrm{O}_{2}$ at 10,21 , and $40 \mathrm{vol} \%$. The experimental uncertainties for mass change were $\leq 0.01 \mathrm{mg}$.

Coupled with a TGA (TA Q5000IR) instrument, an online FTIR spectrometer was used to trace the effluent gas products of a sample. During the measurement, the gas line was maintained at $230^{\circ} \mathrm{C}$, and the spectra were picked up in the range between 4000 and $400 \mathrm{~cm}^{-1}$. Solid products on coal surfaces were traced using an in-situ FTIR spectrometer, equipped with a high temperature reaction chamber, a Bruker IFS 66V/S spectrometer, and a diffuse reflectance accessory (Praying Mantis ${ }^{\mathrm{TM}}$, Harrick). A sample was placed in the reaction chamber, and the gas medium with specific $\mathrm{O}_{2}$ concentration was then introduced into the chamber slowly. A measurement commenced after 30 min of gas environment preparation. The spectra of the solid surface at various temperatures were collected between 4000 and $700 \mathrm{~cm}^{-1}$. These two types of FTIR measurements were both started by heating the samples from the ambient temperature to $400^{\circ} \mathrm{C}$ at $4^{\circ} \mathrm{C} / \mathrm{min}$. During the operation of the spectrometers, the scanning times for both the background and sample were set to 32 at a resolution of $4 \mathrm{~cm}^{-1}$. Differential spectra were generated by means of the software Omnic 7.4 (Thermo Fisher Scientific).

\section{Results and Discussion}

\subsection{Phenomena Observed by Analytical Instrumentations}

The TG/DTA data for the samples either under $\mathrm{N}_{2}$ or aerial atmosphere are shown in Figure 1. As shown by the TG results collected under $\mathrm{N}_{2}$ atmosphere, the sample mass undergoes progressive decrease with the temperature increase. The mass loss becomes significant at temperatures $>350^{\circ} \mathrm{C}$. Negative values of the DTA signals were obtained at the beginning of the measurement, but immediately returned to zero and remained constant throughout the measurement. Under aerial atmosphere, the sample undergoes a slight decline in its mass, which corresponds to the endothermic stage of the DTA signals. Afterwards, the sample mass displays progressive increase, and then reaches the maximum at $\sim 275^{\circ} \mathrm{C}$; meanwhile, the DTA data become positive, and the rate of heat flow reaches its maximum at $\sim 300^{\circ} \mathrm{C}$. As the temperature increases further, the sample mass begins to decrease rapidly, whereas the rate of heat flow exhibits a decline followed by a considerable increase. The trends displayed by the DTA curve for the sample under the

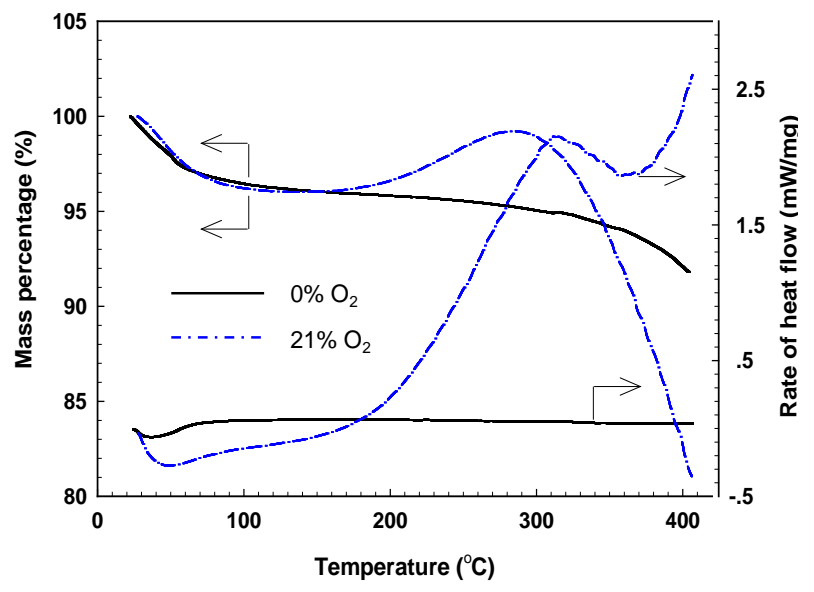

Figure 1. TG and DTA curves obtained by heating the samples either in nitrogen or air at a rate of $4^{\circ} \mathrm{C} / \mathrm{min}$.

aerial atmosphere are consistent with those reported by Banerjee and co-worker [11].

During pyrolysis of a sample in nitrogen, the mass loss at the initial stage is mainly ascribed to the removal of moisture. The progressive mass change between $150^{\circ} \mathrm{C}$ and $300^{\circ} \mathrm{C}$ indicates a low rate of decomposition reactions. At $\sim 350^{\circ} \mathrm{C}$, the decomposition reactions begin to accelerate, resulting in significant mass loss. For the sample under oxidation in air, the initial mass loss was also mainly led by water evaporation, which is demonstrated by the negative values in the rate of heat flow. As a result of oxygen uptake and formation of solid oxygenated complexes, the sample mass starts to increase, and the corresponding rate of heat flow becomes positive. After reaching the maximum at $\sim 275^{\circ} \mathrm{C}$, the sample mass tends to decrease with the further increase in temperature. This is a sign to indicate the acceleration in the rate of thermal decomposition (endothermic) reactions: on one hand, it makes the sample mass decrease; on the other hand, the heat absorbed by the thermal decomposition of unstable oxygenated complexes offsets in part the heat evolved by the oxygen chemisorption, thereby reducing the rate of heat flow. The DTA curve rebounds again after slight decrease, which is assigned to the significant mass loss shown in the TG curve. This phenomenon suggests that another exothermic reaction comes into action at this stage.

The variations of the TG and DTA curves for the sample heated in aerial atmosphere are essentially related to the multiple reactions that occur during the oxidation process. By contrast, the sample heated under nitrogen atmosphere always undergoes thermal decomposition reactions throughout the measurement. The obvious differences shown by these two sets of TG/DTA curves indicate that the reaction medium plays an important role in altering the behavior of a coal sample during heating. 
Li et al. [12] reported their TG/DTA results for oxidation of a bituminous coal and several types of refusederived fuels. The trends of mass change and the cumulative heat for all of the samples observed in the temperature range between $0^{\circ} \mathrm{C}$ and $400^{\circ} \mathrm{C}$ are similar to those described in the present study. Instead of providing detailed analysis on the experimental data, they stated that the progressive increase in mass and the rate of heat flow at temperatures $>120^{\circ} \mathrm{C}$ is due to the reactions induced by oxygen adsorption.

The TG curve obtained under aerial atmosphere was analyzed using the parameters defined by Crelling et al. [5], with three characteristic temperatures found below $400^{\circ} \mathrm{C}$. Given the fluctuation of the derivative TG (DTG) data at temperatures between $120^{\circ} \mathrm{C}$ and $170^{\circ} \mathrm{C}$, the parameter $T_{1}$ falls between $130.1^{\circ} \mathrm{C}$ and $168.0^{\circ} \mathrm{C}$, whereas the parameters $T_{2}$ and $T_{3}$ are evaluated at $238.4^{\circ} \mathrm{C}$ and $276.2^{\circ} \mathrm{C}$, respectively. $T_{3}$ cannot be considered as the "onset temperature of combustion", because the corresponding DTA data did not illustrate a drastic increase at temperatures $>T_{3}$. Direct scanning electron microscope (SEM) images also show no sign of combustion for the sample even after heating to $400^{\circ} \mathrm{C}$ in air.

Gaseous species formed during the coal oxidation process were traced using an online FTIR spectrometer; the absorption intensities observed at various temperatures are reported in Figure 2. It is evident that within the temperature range studied, the gaseous species released are $\mathrm{CO}_{2}\left(2400 \mathrm{~cm}^{-1}-2220 \mathrm{~cm}^{-1}\right)$, CO $\left(2220 \mathrm{~cm}^{-1}\right.$ - $\left.2050 \mathrm{~cm}^{-1}\right)$, and $\mathrm{H}_{2} \mathrm{O}\left(1800 \mathrm{~cm}^{-1}-1400 \mathrm{~cm}^{-1}\right)$ [13] [14]. The absorption peak of $\mathrm{CO}_{2}$ becomes obvious at $\sim 200^{\circ} \mathrm{C}$, and its intensity increases rapidly with temperature increase. As the major gas product, $\mathrm{CO}_{2}$ formation is directly responsible for the sample mass loss at a higher temperature during the TGA measurement.

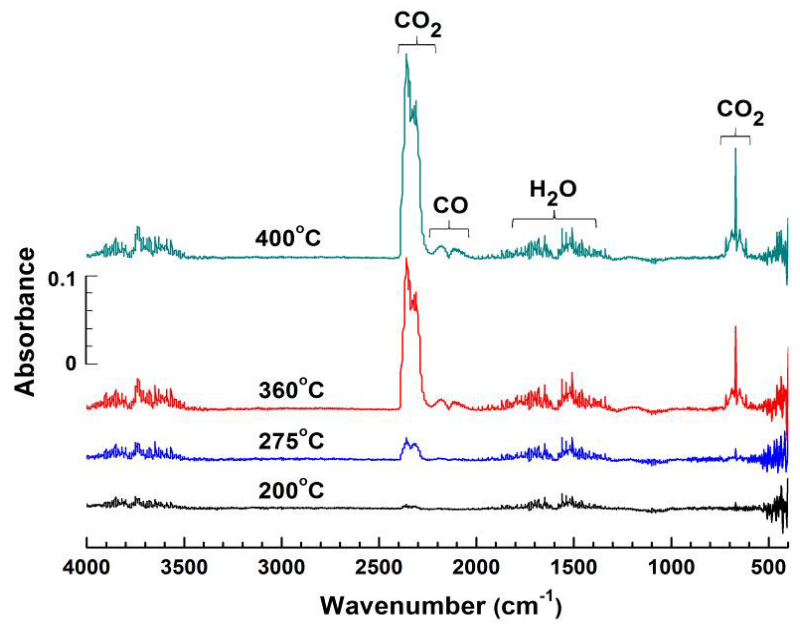

Figure 2. FTIR spectra of gas products evolved at various temperatures for a sample heated at $4^{\circ} \mathrm{C} / \mathrm{min}$ in air.
FTIR spectra of the solid species obtained at various temperatures during the sample oxidation in air are shown in Figure 3. The major absorption peaks and their corresponding species (organic functional groups) were identified and labeled. The observed species usually contain $\mathrm{O}-\mathrm{H}, \mathrm{C}-\mathrm{H}, \mathrm{C}=\mathrm{C}, \mathrm{C}-\mathrm{O}$, and $\mathrm{C}=\mathrm{O}$ bonds [15] [16]. Changes in the functional groups on the sample surfaces were further examined by subtracting FTIR spectra collected at various temperatures (Figure 4). The curve $a$ in Figure 4, which is the subtracted FTIR spectra for a sample at room temperature from those obtained after heating to $275^{\circ} \mathrm{C}$ in nitrogen, indicates that a coal sample mainly experiences the water removal and hydroxyl decomposition in an inert atmosphere [14] [16].

Significant changes have been observed in the major absorption bands for the sample oxidized at $275^{\circ} \mathrm{C}$ (curve

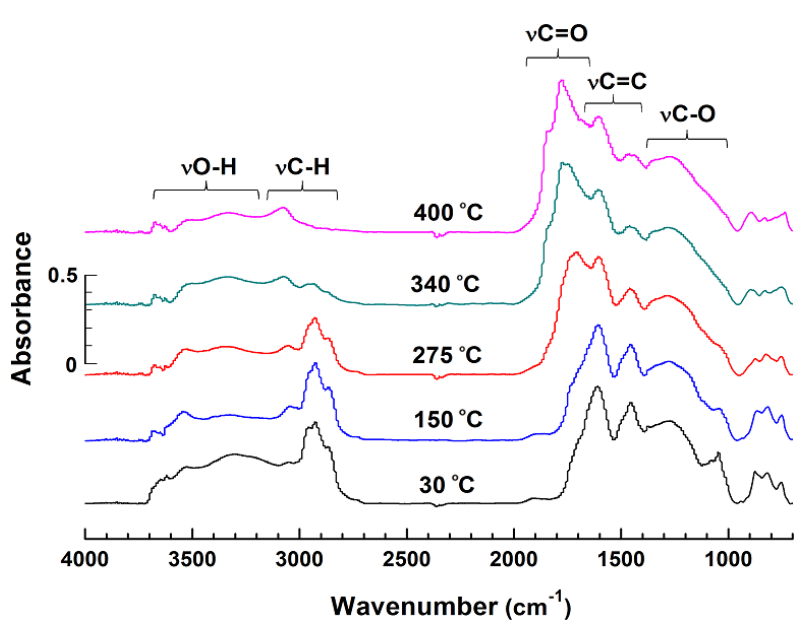

Figure 3. FTIR spectra observed on the surfaces of a sample at various temperatures during heating at $4^{\circ} \mathrm{C} / \mathrm{min}$ in air.

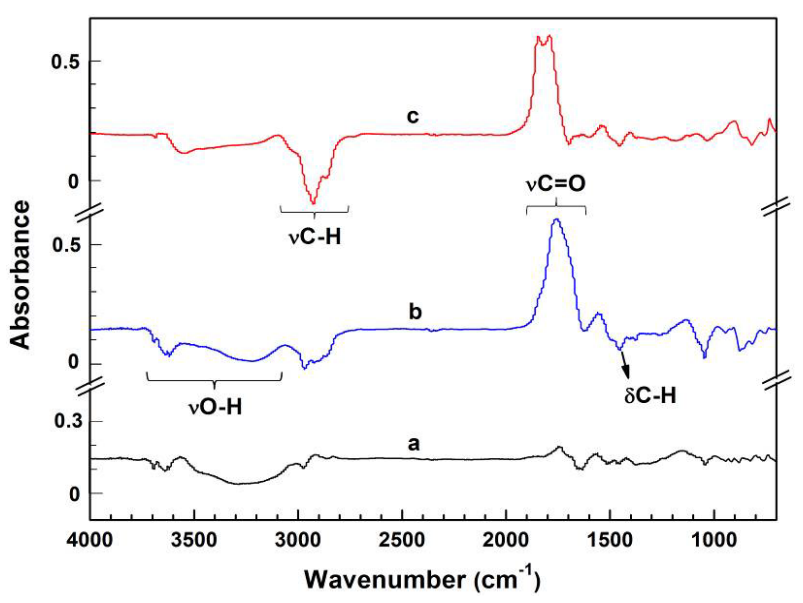

Figure 4. Comparisons of the subtracted FTIR spectra for the samples at ambient temperature from those after heating to $275^{\circ} \mathrm{C}$ either in nitrogen (a) or in air (b), as well as the spectra for the sample oxidized to $275^{\circ} \mathrm{C}$ from those after heating to $400^{\circ} \mathrm{C}$ in air (c). 
$b$ in Figure 4). The weakening of the bands in the region $3680 \mathrm{~cm}^{-1}-3100 \mathrm{~cm}^{-1}$ is also due to water removal and decomposition of hydroxyls. The intensities of the peaks for $v \mathrm{C}-\mathrm{H}$ ( $v$ : stretching vibration) $\left(3050 \mathrm{~cm}^{-1}-2800 \mathrm{~cm}^{-1}\right)$ and $\delta \mathrm{C}-\mathrm{H}$ ( $\delta$ : bending vibration) $\left(1450 \mathrm{~cm}^{-1}\right)$ decrease significantly, indicating loss of hydrocarbon species under the aerial atmosphere [4] [17]. Strong positive absorption peaks centered at $\sim 1760 \mathrm{~cm}^{-1}$ appear, implying the formation of abundant oxygenated complexes containing $\mathrm{C}=\mathrm{O}$ bonds. It is suggested that the oxidation products formed at $\sim 275^{\circ} \mathrm{C}$ are mainly those containing $\mathrm{C}=\mathrm{O}$ bonds, including aldehydes and carboxylic acids.

The subtracted FTIR spectra for a sample oxidized at $275^{\circ} \mathrm{C}$ from those after heating to $400^{\circ} \mathrm{C}$ in air are shown as curve $c$ in Figure 4. The appearance of the negative absorption bands at $3630 \mathrm{~cm}^{-1}-3100 \mathrm{~cm}^{-1}$ for $v \mathrm{O}-\mathrm{H}$ demonstrates that with the further increase in temperature, hydroxyls continue to be condensed or oxidized. Similar to curve $b$ in Figure 4, the negative bands assigned to $v \mathrm{C}-\mathrm{H}\left(3100 \mathrm{~cm}^{-1}-2800 \mathrm{~cm}^{-1}\right)$ and $\delta \mathrm{C}-\mathrm{H}\left(1450 \mathrm{~cm}^{-1}\right)$ are still strong, thereby indicating accelerated rate of oxidation reactions with alkyls. The strong positive absorption peaks of $\mathrm{C}=\mathrm{O}$ at higher bands $\left(2000 \mathrm{~cm}^{-1}-1720 \mathrm{~cm}^{-1}\right)$ mainly belong to the anhydrides and esters [4] [15]. Weak negative absorption bands of $\mathrm{C}=\mathrm{O}$ appear in the region of $1720 \mathrm{~cm}^{-1}-1670 \mathrm{~cm}^{-1}$, corresponding to aldehydes and carboxylic acids. At higher temperatures, more anhydrides and esters are formed as a result of the oxidation of alkyls and the decomposition (or condensation) reactions of carboxylic acids [15] [17]. In addition, the decomposition and condensation reactions among the existing complexes containing $\mathrm{C}=\mathrm{O}$ bonds may cause a loss of certain $\mathrm{C}=\mathrm{O}$ bonds [4] [18].

\subsection{Effect of Oxygen Concentration on the Coal Oxidation}

The TG curves for coal samples oxidized at different oxygen concentrations are compared in Figure 5(a).

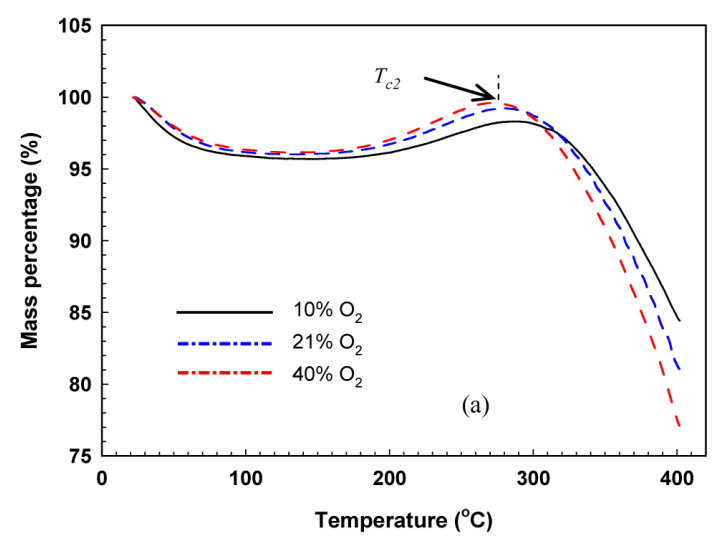

These curves have similar variation patterns, which almost overlap at the initial stage. With the increase in oxygen concentration, the mass increase peak rises, whereas the peak temperature decreases. In the higher temperature region, an increase in oxygen concentration results in more significant decrease in the TG curve, indicating acceleration in the mass loss rate of a sample. The residual mass at the conclusion of the measurements decreases by $\sim 7$ wt\% with an increase of $\mathrm{O}_{2}$ concentration from 10 to 40 vol\%.

As shown in Figure 5(b), the water evaporation process contributes to the variation in the DTA curves at the initial stage. At the stage of mass increase of a sample, the rate of heat flow increases more significantly with the increase in $\mathrm{O}_{2}$ concentration, which corresponds to an increased amount of heat evolved. The rate of heat flow decreases slightly at a temperature exceeding $\sim 300^{\circ} \mathrm{C}$, but rebounds at $\sim 360^{\circ} \mathrm{C}$. The rate increment between $\sim 360^{\circ} \mathrm{C}$ and $400^{\circ} \mathrm{C}$ consistently increases with the increase in $\mathrm{O}_{2}$ concentration.

Several parameters are defined to characterize these TG and DTA curves, including three characteristic temperatures, the percentage of mass increase at $\sim 275^{\circ} \mathrm{C}$, the amount of heat evolved in association with the sample mass increase, and the percentage of increase in the heat flow rate at $400^{\circ} \mathrm{C}$. The parameter $T_{c 1}$ is the temperature of the complete water evaporation, i.e. the starting point at which the rate of heat flow becomes positive. $T_{c 2}$ is the peak temperature at the mass increase stage, whereas $T_{c 3}$ is the second transition temperature of the exothermic peak in the DTA curve (Figure 5(a) \& Figure 5(b)). The percentage of mass increase at $\sim 275^{\circ} \mathrm{C}$ is determined by subtracting the minimum sample mass at $\sim 150^{\circ} \mathrm{C}$ from the maximum mass between $150^{\circ} \mathrm{C}$ and $300^{\circ} \mathrm{C}$, and divided by the minimum sample mass at $\sim 150^{\circ} \mathrm{C}$ [16]. The amount of heat evolved in association with the sample mass increase is obtained by integrating the exothermic peak area of the DTA curve between $T_{c 1}$ and $T_{c 3}$ (Figure 5(b)).

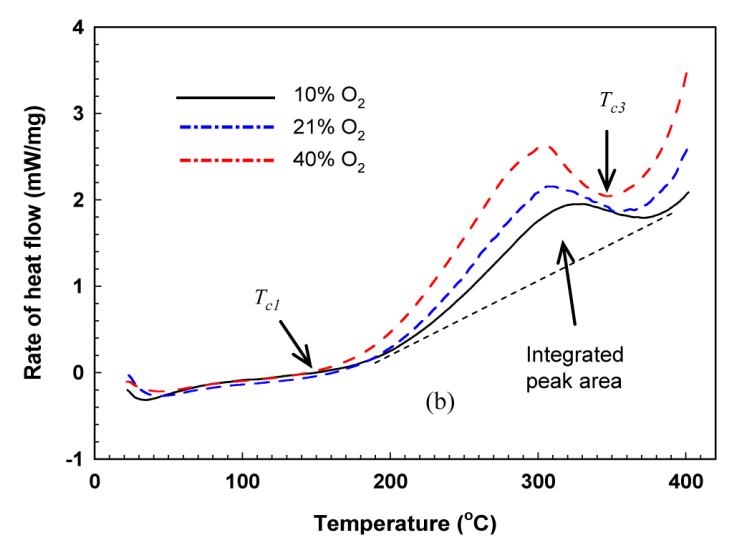

Figure 5. TG (a) and DTA (b) results measured for coal samples oxidized under different oxygen concentrations. 
The percentage of increase in the heat flow rate at $400^{\circ} \mathrm{C}$ is defined by the heat flow rate at $400^{\circ} \mathrm{C}$ minus that at $T_{c 3}$ and then divided by that at $T_{c 3}$. The results of these parameters are given in Table 2.

As shown in Table 2, with the increase in oxygen concentration, $T_{c 2}$ and $T_{c 3}$ decrease, but the percentage of mass increase at $\sim 275^{\circ} \mathrm{C}$, the associated amount of heat evolved, and the percentage of increase in the heat flow rate at $400^{\circ} \mathrm{C}$ increase. For the oxygen concentration varying from $10 \mathrm{vol} \%$ to $21 \mathrm{vol} \%$, the percentage of mass increase grows from $2.74 \%$ to $3.35 \%$ ( $0.61 \%$ increase). However, once the oxygen concentration varies from 21 vol\% to $40 \mathrm{vol} \%$, the extent of increase is only $0.22 \%$. A continuously increasing trend is observed for the associated amount of heat evolved. These results suggest no linear dependence between the percentage of mass increase and the associated amount of heat evolved at varying oxygen concentrations. This is understandable, because the mass increase and the heat release are the comprehensive performance of a series of exothermic and endothermic reactions taking place during the oxidation process.

Temperature-dependent emission of $\mathrm{CO}_{2}$ for the coal samples oxidized under different oxygen concentrations is shown in Figure 6. For a specific measurement, the absorption intensity of $\mathrm{CO}_{2}$ varies in consistency with the corresponding rate of heat flow (refer to Figure 5(b)), and its variations can be split into two distinct phases. The first phase is between $200^{\circ} \mathrm{C}$ and $360^{\circ} \mathrm{C}$ for the sample oxidized in air. With the temperature increase, the intensity of the absorption peak grows progressively, followed by a quasi-steady increase at temperatures $>300^{\circ} \mathrm{C}$. These results somehow reflect the variation trends in the rate of decomposition reactions. In the second phase $\left(\geq 360^{\circ} \mathrm{C}\right.$ ), the rate of $\mathrm{CO}_{2}$ production undergoes more drastic and persistent increase, indicating the commencement of a different mechanism of gas evolution.

Consistency in the patterns of gas evolution at these two consecutive phases has been observed in the measurements at various $\mathrm{O}_{2}$ concentrations. By recalling the variation trends of the DTA signals (Figure 5(b)), we confirm that at temperatures $>\sim 360^{\circ} \mathrm{C}$, the gas products may be sourced by an extra formation mechanism, and oxygen plays a role in both promoting the rate of $\mathrm{CO}_{2}$ formation and the amount of heat evolved. There is no doubt that only the direct "burn-off" reaction serves these purposes.

The compositions on coal surfaces traced during the oxidation under 40 vol\% $\mathrm{O}_{2}$ by using an in-situ FTIR spectrometer are compared to those under $10 \mathrm{vol} \% \mathrm{O}_{2}$ (Figure 7). Curves $a_{1}$ and $a_{2}$ are the subtracted FTIR spectra for the samples at ambient temperature from those after heating to $275^{\circ} \mathrm{C}$. With the increase in $\mathrm{O}_{2}$
Table 2. List of the data for $T_{c 2}$ and $T_{c 3}$, percentage of mass increase at $\sim 275^{\circ} \mathrm{C}$, the amount of heat evolved in association with the sample mass increase, and the percentage of increase in the heat flow rate at $400^{\circ} \mathrm{C}$.

\begin{tabular}{cccccc}
\hline $\begin{array}{c}\text { Oxygen } \\
\text { conc. } \\
(\text { vol\%) }\end{array}$ & $\begin{array}{c}T_{c 2} \\
\left({ }^{\circ} \mathrm{C}\right)\end{array}$ & $\begin{array}{c}T_{c 3} \\
\left({ }^{\circ} \mathrm{C}\right)\end{array}$ & $\begin{array}{c}\text { Perc. of } \\
\text { mass increase } \\
\text { at } 275^{\circ} \mathrm{C} \\
(\%)\end{array}$ & $\begin{array}{c}\text { Amount } \\
\text { of heat } \\
\text { evolved } \\
(\mathrm{kJ} /(\mathrm{kg} \\
\text { raw coal) })\end{array}$ & $\begin{array}{c}\text { Perc. of } \\
\text { increase in } \\
\text { heat flow } \\
\text { rate at } \\
400^{\circ} \mathrm{C}(\%)\end{array}$ \\
\hline 0 & - & - & - & - & - \\
10 & 288.3 & 374.7 & 2.74 & 870 & 16.0 \\
21 & 280.1 & 364.4 & 3.35 & 1040 & 38.9 \\
40 & 272.0 & 347.7 & 3.57 & 1320 & 74.3 \\
\hline
\end{tabular}

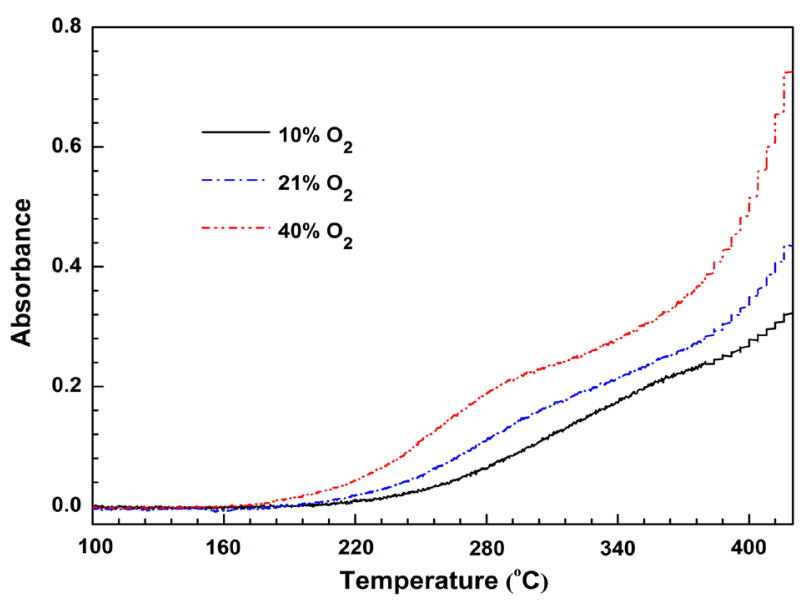

Figure 6. Temperature-dependent FTIR absorbance for $\mathrm{CO}_{2}$ evolved from coal samples oxidized under various oxygen concentrations.

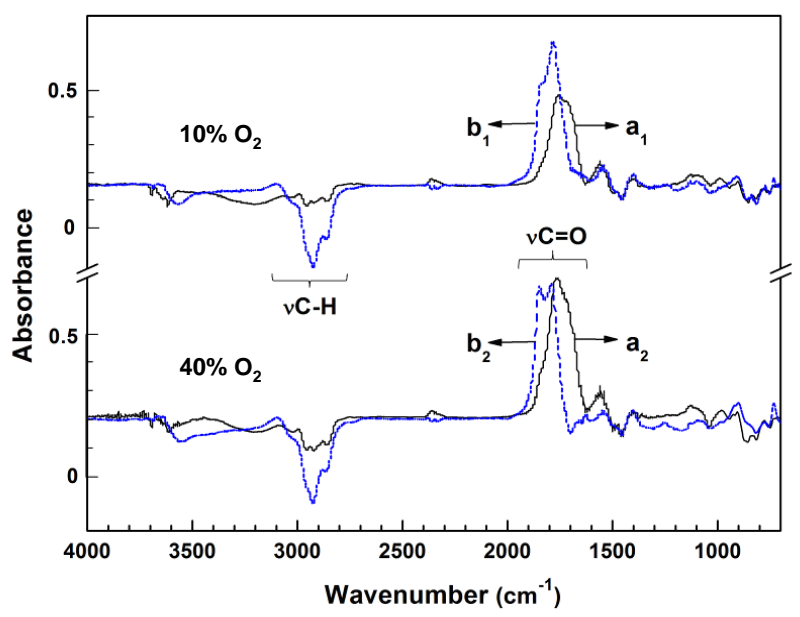

Figure 7. Comparisons of the subtracted FTIR spectra for the samples oxidized in the atmospheres containing $10\left(a_{1} \& b_{1}\right)$ and 40 vol\% $\mathrm{O}_{2}\left(a_{2} \& b_{2}\right)$, respectively. Curves $a_{1} \& a_{2}$ were obtained by subtracting the spectra of the samples at ambient from those after heating to $275^{\circ} \mathrm{C}$ under 10 or $40 \mathrm{vol} \% \mathrm{O}_{2}$, and curves $b_{1} \& b_{2}$ were generated by subtracting the spectra of the samples oxidized to $275^{\circ} \mathrm{C}$ from those after heating to $400^{\circ} \mathrm{C}$. 
concentration from $10 \mathrm{vol} \%$ to $40 \mathrm{vol} \%$, the negative absorption intensity of $v \mathrm{C}-\mathrm{H}$ bands $\left(3100 \mathrm{~cm}^{-1}-2800\right.$ $\mathrm{cm}^{-1}$ ) increases; meanwhile, the positive absorption intensity of $v \mathrm{C}=\mathrm{O}$ bands $\left(1880 \mathrm{~cm}^{-1}-1650 \mathrm{~cm}^{-1}\right)$ becomes stronger. These results demonstrate that with the increase in the oxygen concentration, greater amount of $\mathrm{O}_{2}$ is adsorbed, and more hydrocarbon species are oxidized. As a result, extra amounts of aldehydes and carboxylic acids are formed in the coal structure.

The phenomena observed above correspond to the following reaction steps:

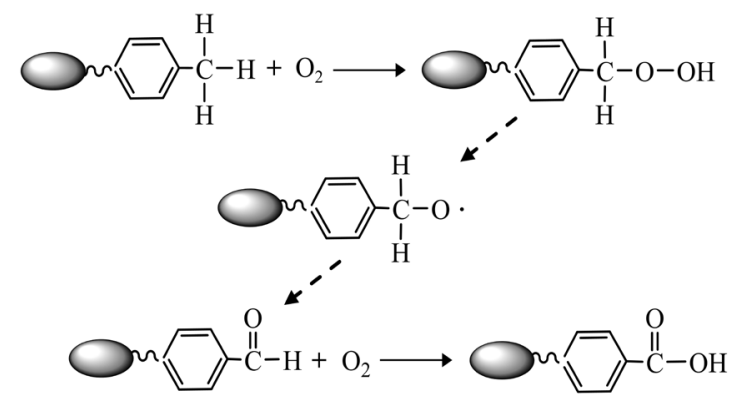

where Reaction 4 contains a few sub-steps in principal, including the activation of carbonyl species by chain carriers (H-O) and the subsequent formation of peroxides at the carbon centers [2] [4] [8].

It is evident that an increase in the oxygen concentration speeds up the rates of these two reactions. This has been reflected by an increase in the percentage of mass increase at $\sim 275^{\circ} \mathrm{C}$. The increased rates of these reactions implies an increase in the amount of heat released, i.e. the enlarged exothermic peak area on the individual DTA curves (refer to Figure 5(b) and Table 2).

Obvious differences are also observed between the subtracted FTIR spectra for the samples oxidized at $275^{\circ} \mathrm{C}$ and those after heating to $400^{\circ} \mathrm{C}$, for example, curve $a_{2}$ versus $b_{2}$ in Figure 7. Stronger absorption peaks of $v \mathrm{C}-\mathrm{H}$ bands are observed in the region of $3100 \mathrm{~cm}^{-1}$ $2800 \mathrm{~cm}^{-1}$, implying that larger amount of hydrocarbon species is consumed at higher temperatures. Unlike the phenomena illustrated by the curves $a_{1}$ and $b_{1}$, the absorption intensities of $v \mathrm{C}=\mathrm{O}$ bonds are almost identical for the sample oxidized to $275^{\circ} \mathrm{C}$ and $400^{\circ} \mathrm{C}$ under $40 \mathrm{vol} \%$ $\mathrm{O}_{2}$. These results indicate that at $\sim 400^{\circ} \mathrm{C}$, more hydrocarbon species in coal structure are consumed via direct "burn-off" reaction, rather than the chemisorption sequence. This is also an evidence to demonstrate that an increase in oxygen concentration may speed up the direct "burn-off" reaction, which explains the obvious reduction in the residual mass at the end of the TGA measurement in an oxygen-enriched atmosphere.

Following the above statements, it is straightforward to establish that the considerable difference in mass loss at the end of the measurements for the samples oxidized in either an oxygen-leaned or oxygen-enriched environment is essentially contributed by the direct "burn-off" reaction, rather than the chemisorption sequence. This happens only when a shift of the governing reactions is performed from the chemisorption sequence, which is primarily operated at temperatures below $360^{\circ} \mathrm{C}$, to the direct "burn-off" reaction at the temperatures above $360^{\circ} \mathrm{C}$.

\subsection{Characterization of the Oxidation Process at Different Temperature Ranges}

As demonstrated by the TG and DTA results, identical trends are exhibited for the samples oxidized in various oxygen concentrations (Figure 5). Comparable patterns of the mass change and heat evolution have been reported in the literature for various types of coals and biomass using the same experimental approach [6] [7] [12]. Thus, TG/DTA curves are used to characterize the coal oxidation process at different temperature ranges. Based on the TG/DTA curves obtained by oxidation measurement in air, the oxidation process can be divided into the following typical stages: 1$)$ water evaporation $(T \leq$ $\left.T_{c 1}\right)$, 2) oxygen chemisorption $\left(T_{c 1}<T \leq T_{c 2}\right), 3$ ) significant decomposition reactions $\left(T_{c 2}<T \leq T_{c 3}\right)$, and 4) the stage dominated by the direct "burn-off" reaction $\left(T>T_{c 3}\right)$ (Figure 8).

As is well known, coal contains certain amount of water, and during its oxidation below $T_{c 1}$, the exothermic oxidation reactions and endothermic water evaporation coexist. Given that the amount of heat released by the former is smaller than that absorbed by the later, negative values are presented on the DTA curve at this stage.

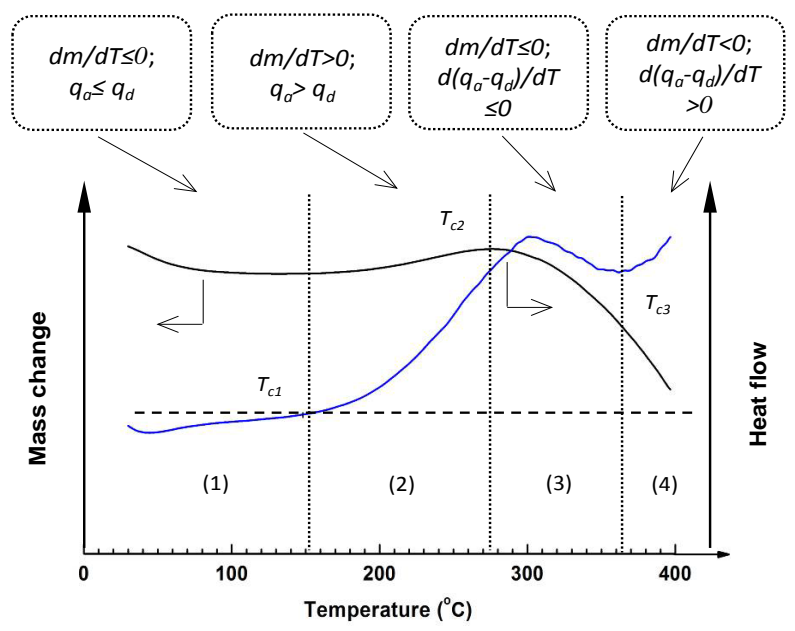

Figure 8. Four stages of coal oxidation up to $400^{\circ} \mathrm{C}$ determined by a set of TG/DTA curves: (1) Water evaporation; (2) Oxygen chemisorption; (3) Significant decomposition reactions; and (4) The stage dominated by direct "burn-off" reaction. 
Meanwhile, the mass loss led by water evaporation is much larger than the mass increase due to oxygen adsorption, thereby resulting in the observed decline in the TG curve.

At the stage of oxygen chemisorption, the internal coal pores begin to expose to the oxidation medium because of water removal; hence, the rate of chemisorption increases. The mass increase of a sample reaches the maximum at $\sim 275^{\circ} \mathrm{C}$. At this stage, the decomposition of unstable oxygenated complexes does occur, and the amount of heat evolved is the compromised result of the instantaneous heat of exothermic chemisorption reactions $\left(q_{a}\right)$ and that of endothermic decomposition reactions $\left(q_{d}\right)$. Given that thermal decomposition of unstable oxygennated complexes is a relatively slow process at temperatures between $T_{c 1}$ and $T_{c 2}$, the amount of heat released increases with the temperature rise, which has been displayed by the DTA curves. The associated infrared spectra for solid products also clearly show that these phenomena are primarily contributed by the chemisorption of $\mathrm{O}_{2}$ and the formation of the species containing $\mathrm{C}=\mathrm{O}$ bonds, as a result of interaction of the active groups in coal structure with $\mathrm{O}_{2}$.

Significant decomposition reactions occur at temperatures between $T_{c 2}$ and $T_{c 3}$, and the rate of heat evolution by oxygen adsorption is not enough to offset that consumed by the thermal decomposition reactions in general, i.e. $\mathrm{d}\left(q_{a}-q_{d}\right) / \mathrm{d} T \leq 0$. Therefore, the amount of heat released $\left(q_{a}-q_{d}\right)$ reduces, which is illustrated by a decline in the rate of heat flow. Accordingly, the sample mass decreases because of the accelerated decomposition reactions (refer to Figure 5 \& Figure 6). These results show that the oxygenated complexes containing $\mathrm{C}=\mathrm{O}$ bonds are relatively stable below $T_{c 2}\left(<300^{\circ} \mathrm{C}\right)$, whereas obvious decomposition occurs at temperatures $\geq 300^{\circ} \mathrm{C}$, resulting in a reduction of the total amount of heat released. For this reason, $T_{c 2}$ can be treated as the critical temperature at which a considerable amount of oxygenated complexes begin to decompose. This understanding is consistent with the statements of Berkowitz [19] regarding the stability of humic acids that contain such functional groups.

As the temperature increases further $\left(>T_{c 3}\right)$, the "burnoff" reaction is remarkably accelerated, which contributes to the drastic decline in the TG curves and a rapid increase in the DTA signals. Consequently, the stage of significant decomposition reactions is replaced by the stage dominated by the "burn-off" reaction. Worthy of mentioning, a coal sample at this stage is definitely not at the "real" status of combustion or burning, which has been verified by SEM observations on the sample collected after heating to $400^{\circ} \mathrm{C}$ in air.

In the literature [5], the characteristic temperatures are determined solely based on TG and the DTG data. As a result, these characteristic temperatures have no direct link to chemical reactions and the associated heat release in coal oxidation. The definition of these characteristic temperatures is also questionable. Parameter $T_{1}$ is named as the "temperature of initial chemisorption", which is the first transition point at which the TG curve shifts from mass loss to mass gain. In fact, chemisorption starts from the beginning of a test, even though the mass gain by $\mathrm{O}_{2}$ adsorption is concealed by the mass loss because of water evaporation below $T_{1}$.

Crelling et al. [5] defined the parameter $T_{2}$ as the "temperature of maximum rate of chemisorptions", i.e., the first peak temperature on a DTG curve. The oxidation of coal is not a simple oxygen adsorption process, and the phenomenon of mass increase is the overall reflection of a series of reactions leading to mass gain and loss; therefore, we are unable to expect a direct link between the maximum rate of chemisorptions and the maximum rate of mass increase. The "temperature of combustion onset", $T_{3}$, is actually the temperature at which a sample mass reaches its maximum, which is equivalent to $T_{c 2}$ in the present study. There is no doubt that the characteristic temperatures introduced in the present study have clear physical significance and solid chemistry basis.

Once the rank of coal is very high, its volatile matter content becomes low, which implies a smaller amount of $\mathrm{C}-\mathrm{H}$ bonds stored in the coal structure and fewer carbon centers available for the oxygen chemisorption reactions. As a result, the stage of significant decomposition reactions tends to be limited, which can be seen from the typical DTA or differential scanning calorimeter (DSC) results for the semi-anthracite or anthracite [20] [21]. As the carbon content is maintained at $\sim 90 \%$, the decline segment on the DSC curve (at the temperatures usually $\leq T_{c 3}$ ) may disappear. Under this circumstance, the stage of significant decomposition reactions is then replaced by that dominated by the direct "burn-off" reaction.

The consistent decrease in the values of $T_{c 2}$ and $T_{c 3}$ for the samples oxidized under the oxygen concentrations from 10 to $40 \mathrm{vol} \%$ is an indication of the role of $\mathrm{O}_{2}$ molecules in altering the oxidation process. The presence of $\mathrm{O}_{2}$ triggers the formation of the transient intermediates (Reaction 3) and their further oxidation into aldehydes and other oxygenated complexes (e.g., Reaction 4). An increase in oxygen concentration implies the acceleration in the rates of oxygen chemisorption and formation of solid oxygenated complexes, leading to a growth in both the percentage of mass increase at $\sim 275^{\circ} \mathrm{C}$ and the associated amount of heat evolved. In addition, enrichment of $\mathrm{O}_{2}$ in the reaction medium indirectly intensifies the decomposition reactions of the oxygenated complexes and speeds up the "burn-off" reaction. As a result, the corresponding decrease in characteristic tempera- 
tures $T_{c 2}$ and $T_{c 3}$ was observed.

Shift in the governing reactions at various temperatures is an overall reflection of the temperature effect on these reactions, which are particularly due to their diverse requirements of reactants and the energy barriers to overcome. Desorption of water requires overcoming the minimum energy barriers and makes it available for $\mathrm{O}_{2}$ molecules to access the unveiled active carbon centers in coal pores [22]. Because of the lower energy barriers for oxygen adsorption (both physical and chemical), chemisorption of oxygen proceeds relatively easily compared with the direct "burn-off" reaction, especially at low temperatures [2] [8]. High temperatures $\left(>360^{\circ} \mathrm{C}\right)$ and the deformed coal structure, which is a consequence of the significant decomposition reactions, provide both energy and material basis for rapid progress in the direct "burnoff" reaction.

In engineering practice, coal may not be always exposed to a flowing environment, and when water is removed to a certain extent, the remaining water could act as a catalyst to the oxygen chemisorption reactions [2]. Oxygen chemisorption reactions provide initial energy for the self-heating of coal in the absence of external heat sources. When the temperature is high enough, the coal eventually moves toward spontaneous combustion because of the significant involvement of the direct "burnoff" reaction. The self-heating and spontaneous combustion phenomena of coal not only correspond to two distinct stages in the oxidation process, but also are controlled by these two major reactions, respectively. From this standpoint, in the assessment on the propensity of a coal towards self-heating and spontaneous combustion, appropriate methods should be used to focus on either the oxygen adsorption capacity or the capability of coal to participate in the direct "burn-off" reaction.

\section{Concluding Remarks}

During the oxidation at $<\sim 275^{\circ} \mathrm{C}$, the alkyl chains in coal structure are converted into $\mathrm{C}=\mathrm{O}$ containing species, leading to the increase in sample mass and the associated amount of heat evolution. At temperatures $\sim 400^{\circ} \mathrm{C}$, the direct "burn-off" reaction of hydrocarbon species, which is responsible for the higher rate of $\mathrm{CO}_{2}$ emission of a sample, becomes dominated.

An increase in oxygen concentration in the reaction medium results in an increased amount of heat evolved and a decreased temperature threshold for initiating significant thermal decomposition reactions. The presence of sufficient oxygen speeds up the oxidation process by primarily promoting oxygen chemisorption, formation and decomposition of the solid complexes containing $\mathrm{C}=\mathrm{O}$ groups, as well as the direct "burn-off" reaction.
The products both in gas and solid phase, as well as the heat evolution allow the coal to demonstrate distinct characteristics during its oxidation at varying temperatures. With the temperature increase, the oxidation process can be classified into four typical stages, namely, water evaporation, oxygen chemisorption, significant decomposition reactions, and the stage dominated by the direct "burn-off" reaction. Temperature boundaries of these stages can be determined using the parameters defined based on a set of TG/DTA data.

\section{Acknowledgements}

In situ FTIR measurements on solid surfaces were performed at the infrared and far-infrared beamline station of National Synchrotron Radiation Laboratory (NSRL) in Hefei, China. We would like to thank Assoc. Prof. Zeming Qi and his colleagues for providing assistance during the measurements.

\section{REFERENCES}

[1] van Krevelen, D.W. (1993) Coal: Typology-Chemistry-Physics-Constitution. 3rd Edition, Elsevier, New York, 627-658.

[2] Wang, H.-H., Dlugogorski, B.Z. and Kennedy, E.M. (2003) Coal Oxidation at Low Temperatures: Oxygen Consumption, Oxidation Products, Reaction Mechanism and Kinetic Modeling. Progress in Energy and Combustion Science, 29, 487-513. http://dx.doi.org/10.1016/S0360-1285(03)00042-X

[3] Carpenter, D.L. and Giddings, D.G. (1964) The Initial Stages of the Oxidation of Coal with Molecular Oxygen. I. Effect of Time, Temperature and Coal Rank on Rate of Oxygen Consumption. Fuel, 43, 247-266.

[4] Clemens, A.H., Matheson, T.W. and Rogers, D.E. (1991) Low Temperature Oxidation Studies of Dried New Zealand Coals. Fuel, 70, 215-221. http://dx.doi.org/10.1016/0016-2361(91)90155-4

[5] Crelling, J.C., Hippo, E.J., Woerner, B.A. and West Jr., D.P. (1992) Combustion Characteristics of Selected Whole Coals and Macerals. Fuel, 71, 151-158. http://dx.doi.org/10.1016/0016-2361(92)90003-7

[6] He, Q.-L. and Wang, D.-M. (2005) Comprehensive Study on Coal Oxidation Process by TG-DTA-FTIR Techniques. Journal of China Coal Society, 30, 53-57. (in Chinese)

[7] Wang, J.-R., Deng, C.-B., Shan, Y.-F., Hong, L. and Lu, W.-D. (2008) A New Method for Classifying Tendency of Coals to Spontaneous Combustion. Journal of China Coal Society, 33, 47-50. (in Chinese)

[8] Wang, H.-H., Dlugogorski, B.Z. and Kennedy, E.M. (2003) Analysis of the Mechanism of the Low-Temperature Oxidation of Coal. Combustion and Flame, 134, 107117. http://dx.doi.org/10.1016/S0010-2180(03)00086-5

[9] Krishnaswamy, S.K., Gunn, R.D. and Agarwal, P.K. (1996) Low-Temperature Oxidation of Coal. 2. An Expe- 
rimental and Modeling Investigation Using a Fixed-Bed Isothermal Flow Reactor. Fuel, 75, 344-352. http://dx.doi.org/10.1016/0016-2361(95)00177-8

[10] Wang, H.-H., Dlugogorski, B.Z. and Kennedy, E.M. (1999) Theoretical Analysis of Reaction Regimes in LowTemperature Oxidation of Coal. Fuel, 78, 1073-1081. http://dx.doi.org/10.1016/S0016-2361(99)00016-2

[11] Banerjee, S.C. and Chakravorty, R.N. (1967) Use of DTA in the Study of Spontaneous Combustion of Coal. Journal of Mines, Metals and Fuels, 15, 1-5.

[12] Li, X.-R., Lim, W.-S., Yusaku, I. and Hiroshi, K. (2009) Safety Evaluation of Sewage-Sludge-Derived Fuels by Comparison with Other Fuels. Fire and Materials, 33, 187-200. http://dx.doi.org/10.1002/fam.991

[13] Carangelo, R.M., Solomon, P.R. and Gerson, D.J. (1987) Application of TG-FT i.r. to Study Hydrocarbon Structure and Kinetics. Fuel, 66, 960-967. http://dx.doi.org/10.1016/0016-2361(87)90336-X

[14] MacPhee, J.A., Giroux, L., Charland, J.-P., Gransden, J.F. and Price, J.T. (2004) Detection of Natural Oxidation of Coking Coal by TG-FTIR-Mechanistic Implications. Fuel, 83, 1855-1860. http://dx.doi.org/10.1016/j.fuel.2004.02.017

[15] Rose, H.R., Smith, D.R. and Vassallo, A.M. (1998) Study of the Oxidation of Oil Shale and Kerogen by Fourier Transform Infrared Emission Spectroscopy. Energy and Fuels, 12, 682-688. http://dx.doi.org/10.1021/ef9701908

[16] Zhan, J., Wang, H.-H., Song, S.-N., Hu, Y. and Li, J.
(2011) Role of an Additive in Retarding Coal Oxidation at Moderate Temperatures. Proceedings of the Combustion Institute, 33, 2515-2522. http://dx.doi.org/10.1016/j.proci.2010.06.046

[17] Iglesias, M.J., de la Puente, G., Fuente, E. and Pis, J.J. (1998) Compositional and Structural Changes during Aerial Oxidation of Coal and Their Relations with Technological Properties. Vibrational Spectroscopy, 17, 41-52. http://dx.doi.org/10.1016/S0924-2031(98)00017-4

[18] Calemma, V., Rausa, R., Margarit, R. and Girardi, E. (1988) FT-i.r. Study of Coal Oxidation at Low Temperature. Fuel, 67, 764-770. http://dx.doi.org/10.1016/0016-2361(88)90147-0

[19] Berkowitz, N. (1979) An Introduction to Coal Technology. Elsevier, New York, 95-130.

[20] Pis, J.J., de la Puente, G., Fuente, E., Morán, A. and Rubiera, F. (1996) A Study of the Self-Heating of Fresh and Oxidized Coals by Differential Thermal Analysis. Thermochimica Acta, 279, 93-101. http://dx.doi.org/10.1016/S0040-6031(96)90066-0

[21] Fangxian, L., Shizong, L. and Youzhi, C. (2009) Thermal Analysis Study of the Effect of Coal-Burning Additives on Combustion of Coals. Journal of Thermal Analysis and Calorimetry, 95, 633-638. http://dx.doi.org/10.1007/s10973-008-9124-x

[22] Wang, H.-H. (2007) Kinetic Analysis of Dehydration of a Bituminous Coal Using TGA Technique. Energy and $\mathrm{Fu}$ els, 21, 3070-3075. http://dx.doi.org/10.1021/ef070170y 